Beat Meier

\title{
150 Jahre Schweizer Pharmakopöe - von lokalen Regelungen zum europäischen Gesetzeswerk
}

Der erste Prozess in der Entwicklung der Pharmakopöen war der Schritt von lokalen und regionalen Vorschriften und Rezeptsammlungen hin zum national verbindlichen Werk. In der Schweiz verlief - bedingt durch die kantonale Hoheit im Gesundheitswesen - diese Phase nicht durchwegs harmonisch. Immerhin war man 1908 so weit, dass die Schweizerische Pharmakopöe für das ganze Land seine Gültigkeit erhielt. Damals wurde die von der 1897 gegründeten Eidgenössischen Pharmakopöekommission erarbeitete, vierte Ausgabe eingeführt. 1936 trat dann die fünfte Ausgabe in Kraft - sie enthielt 1050 Monographien und legte die Nomenklatur für die Arzneistoffe und Arzneimittel verbindlich fest. Die Gefässe in Apotheken und Drogerien mussten entsprechend neu beschriftet werden - auf die Bezeichnung «Ph. Helv. V» stösst der aufmerksame Beobachter auch heute noch häufig.

Die nationale Phase hatte mit der im Ringbuchsystem aufgelegten sechsten Ausgabe der Schweizer Pharmakopöe ihr teilweises Ende: Die steigenden Anforderungen an die pharmazeutische Industrie machten internationale Standards erforderlich. Entsprechend wurde die Zeit reif für eine Europäische Pharmakopöe. Die dazu notwendige Organisation wurde 1964 gegründet. Vorletztes Jahr konnte sie in Strassburg ihr 50-jähriges Bestehen feiern. Die nationalen Monographien wurden sukzessive durch europäische ersetzt - dank dem Loseblattsystem war ein kontinuierlicher Austausch möglich. Heute dominiert

Im Jahr 1865 erschien in der Schweiz erstmals eine nationale Pharmakopöe. Die Pharmacopoea Helvetica Editio Prima war damals noch in lateinischer Sprache verfasst. Swissmedic feierte dieses Jubiläum im Oktober 2015 mit einer Veranstaltung in Bern (Abb. 1, 2).

die Europäische Pharmakopöe; von den Ringbüchern ist man wieder abgekommen. Umso bedeutender sind heute die elektronischen Versionen. Diese haben allerdings das gedruckte Buch lediglich ergänzt und nicht ersetzt. Die Europäische Pharmakopöe erscheint in englischer und französischer Version. Sie wird auch auf Deutsch übersetzt. Die Schweizerische Pharmakopöe wird in den drei Amtssprachen angeboten.

Die Europäische Pharmakopöe wird unter der Regie des EDQM (European Directorate for the Quality of Medicines \& Health Care) entwickelt. Dieses ist dem Europarat angeschlossen - die Schweiz war von Beginn weg an der Ausarbeitung der Monographien mitbeteiligt. Die Schweiz spielt unter den 37 an der Ph. Eur. beteiligten Ländern eine herausragende Rolle, denn $11 \%$ der Experten in den verschiedenen Experten- und Arbeitsgruppen stammen aus der Schweiz, die auch überdurchschnittlich viele Vorsitzende in diesen Gremien stellt. Susanne Keitel, Direktorin des EDQM, lobte dann auch in ihrem Grusswort an der Veranstaltung die Schweiz für ihr Engagement: «Die Zusammenarbeit mit der Schweiz ist für das EDQM ein Traum.» Entsprechend gross sind die Gestaltungsmöglichkeiten der Schweiz, die auf viele Entwicklungen entscheidend Einfluss nehmen kann.
Abb. 1. EDQM-Direktorin Dr. Susanne Keitel überreicht dem Leiter der Abteilung Pharmakopöe bei Swissmedic, Dr. Tobias Gosdschan, zum 150-jährigen Bestehen der Pharmakopöe eine Anerkennung.

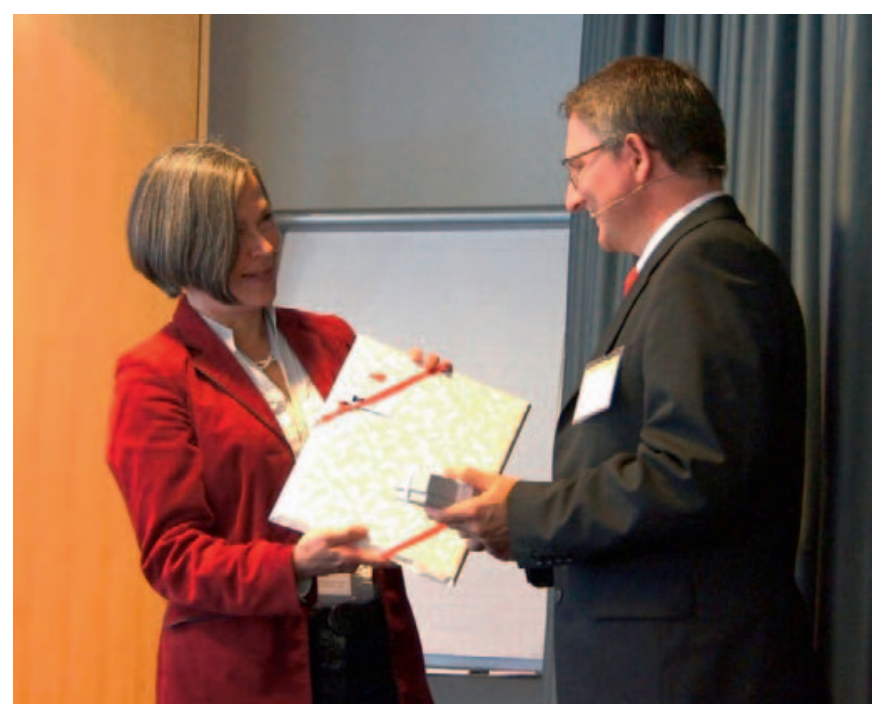




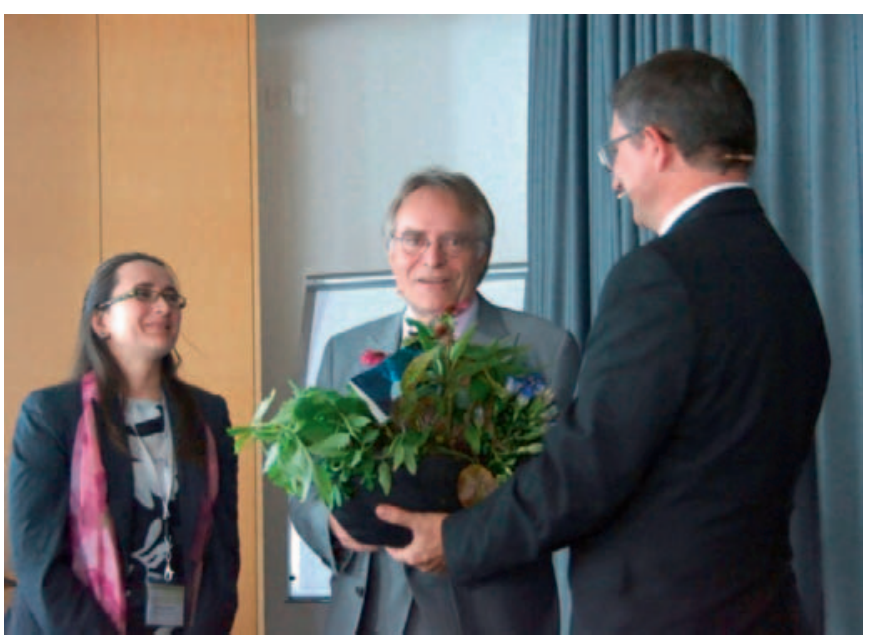

Das Schweizer Engagement bei der Europäischen Pharmakopöe straft die landläufige Meinung Lügen, dass ein kleines Land im Konzert der Grossen nichts bewegen könnte.

Gefeiert wurde jedoch in Bern nicht die Europäische, sondern die Schweizerische Pharmakopöe. Für nationale Bedürfnisse blieb diese bestehen und wird - zusammen mit dem Schweizer Beitrag zur Europäischen Pharmakopöe - unter dem Dach der Swissmedic in einer eigenen Abteilung betreut. Obwohl sie volumenmässig gegenüber der europäischen Ausgabe nur noch klein daherkommt, gibt es Regelungen, die wegweisend sind. Die «Regeln der Guten Herstellungspraxis für Arzneimittel in kleinen Mengen» (Kapitel 20 und 21 der Ph. Helv.) erlauben es Apotheken (auch in den Spitälern), sogenannte Formula-Arzneimittel in kleinen Mengen unter adäquaten pharmazeutischen Bedingungen herzustellen, ohne dass sie auf die umfangreichen, für die Industrie gedachten Regulierungen zurückgreifen müssen. Das Thema wurde vor Kurzem um das Kapitel 20.2 «Regeln der Guten Herstellungspraxis für sterile Arzneimittel in kleinen Mengen» erweitert - diese Vorschriften haben in Europa Modellcharakter.

Gab es zu Beginn der Europäischen Pharmakopöe die Befürchtung, dass die Arzneipflanzen sukzessive aus dem Arzneibuch verschwinden würden, zeigte die Entwicklung das Ge- halten. Monographien für Drogen der Traditionellen Chinesischen Medizin werden erarbeitet, und Herstellungsverfahren und Qualitätsanforderungen der homöopathischen Herstellung fanden Eingang in die Europäische Pharmakopöe. Diese Ausweitung erforderte die Gründung eines Fachausschusses für komplementärmedizinische Arzneimittel in der Schweiz. Dieser erarbeitete in einem grösseren Projekt eine allgemeine Monographie $\mathrm{zu}$ «Anthroposophischen Zubereitungen» und in der Folge das Kapitel «17.7 Herstellungsmethoden für anthroposophische Zubereitungen». Auch das ist ein Beispiel dafür, dass die Schweizerische Pharmakopöe mit neuen Strategien eine Vorreiterrolle übernehmen kann. Darüber berichtete an der Jubiläumstagung Monika Mennet-von Eiff von der Weleda AG. Sie ist die Schweizer Delegierte in der «Homeopathic Raw Materials and Stocks Working Party (HOM)» in Strassburg.

Gab es anfänglich in der Schweizer Pharmakopöekommission noch Widerstand und Skepsis gegenüber komplementärmedizinischen Regelungen, so werden heute diese Bedürfnisse akzeptiert und als Notwendigkeit erachtet. Für die Komplementärmedizin ist es von höchster Bedeutung, in diesem Gesetzeswerk vertreten zu sein, um einerseits über anerkannte Arzneimittel zu verfügen und andererseits zu zeigen, dass auch diese Arzneimittel nach dem Stand der aktuellen pharmazeutischen Herstelltechnik produziert werden. Die allgemeinen Monographien der Arzneibücher zur Herstellung von Arzneimitteln gelten nämlich überall dort, wo sie anwendbar sind, also auch für die Herstellung komplementärmedizinischer Produkte.

Präparatemonographien hatten bei der Entstehung der Arzneibücher einen hohen Stellenwert. Mit der Entwicklung der industriellen Herstellung von Arzneimitteln verlagerten sich die Schwerpunkte zu Wirk- und Hilfsstoffmonographien sowie zu allgemeinen Vorschriften zur Qualität 
und zu Eigenschaften von Arzneiformen. Eine Trendwende ist jedoch nun in Sicht: Viele wertvolle Arzneimittel drohen verloren $\mathrm{zu}$ gehen, weil sie nicht mehr mit genügend Profit im Markt bestehen können. Ein Auffanggefäss ist die Pharmakopöe, die mit Präparatemonographien die Herstellung spezieller Arzneimittel vereinfacht. Die Erarbeitung solcher Monographien ist allerdings mit viel Arbeit verbunden; insbesondere muss die
Stabilität gewährleistet sein. Einige wenige phytotherapeutische Präparatemonographien sind in der Schweizerischen Pharmakopöe erhalten geblieben. Der Fachausschuss Phytochemie modernisiert derzeit die Monographien für Thymiansirup (CH61) und für den zusammengesetzten Feigensirup mit Senna (CH273). Noch gibt es keine Pläne für neue Präparate der Phytotherapie oder der Komplementärmedizin, doch die
Option stünde für weitere Zubereitungen offen.

Die Bedeutung der Pharmakopöe wurde am Jubiläumssymposium deutlich. Der offizielle Auftrag, die neuesten wissenschaftlichen Erkenntnisse in das Gesetzeswerk einfliessen zu lassen, sorgt für ständigen Reformbedarf; neue Wirk- und Hilfsstoffe wollen erfasst sein. So bleibt das Engagement für die Pharmakopöe ein spannender Auftrag für alle beteiligten Kreise. 